\title{
The Expansion of the Periodic Table to its Natural Limits
}

\author{
Andreas Türler
}

\begin{abstract}
One hundred and fifty years after its conception the periodic table of the elements contains 118 members with the $7^{\text {th }}$ period being completely filled. This raises the question of what are the natural limits of the periodic table and whether yet undiscovered elements can be synthesized. Nowadays the alchemists' dream of producing new elements from already known ones has become reality. However, only single, short-lived atoms can be produced and investigated. The current article will give insights into the state of the art concerning the synthesis and chemical investigation of heavy and superheavy elements and discuss limits to the further expansion of the periodic table to even heavier elements in the $8^{\text {th }}$ period.
\end{abstract}

Keywords: Heavy element synthesis · Periodic table $\cdot$ Single atom chemistry $\cdot$ Superheavy elements · Transactinides

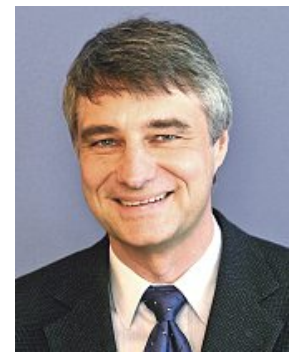

Andreas Türler has been professor of radiochemistry at University of Bern, Switzerland since 2009. He received his diploma and $\mathrm{PhD}$ at University of Bern having carried out research on nucleon transfer reactions under the supervision of Prof. Hans-Rudolph von Gunten. He then joined the group of Prof. Darleane C. Hoffman at Lawrence Berkeley National Laboratory, Berkeley, US, as postdoctoral fellow. In 1992, he moved back to Switzerland and in the following years worked as staff scientist at PSI, Villigen. His habilitation in 2000 at University of Bern was followed by an appointment as full professor and director of the Institute of Radiochemistry at TU Munich, Germany from 2001 to 2009.

\section{The Periodic Table of the Elements Today}

One hundred and fifty years after its conception, the Periodic Table (PT) is today, for once, complete and without gaps, with a fully filled $7^{\text {th }}$ row (Fig. 1) and no known element beginning the $8^{\text {th }}$ row. The recent discovery of elements with atomic numbers $113,115,117$, and 118 has been validated by a joint working group of the International Union of Pure and Applied Chemistry (IUPAC) and the International Union of Pure and Applied Physics (IUPAP). Assignation of priority of discovery to a laboratory or to a collaborating group of laboratories has been made. ${ }^{[1,2]}$ The synthesis and detection of heavy and superheavy elements will be discussed in detail in section 2 of this review. The names and chemical symbols proposed by the discoverers were accepted by the IUPAC Inorganic Chemistry Division in accordance with IUPAC recommendations on how to name a new element. ${ }^{[3,4]}$ The most important adjustment was that the names of new elements should have an ending that "reflects and maintains historical and chemical consistency. This would be in general "-ium" for elements belonging to groups $1-16$, i.e. including the f-block elements, "-ine" for elements of group 17 and "-on" for elements of group 18.". ${ }^{[4]}$ The positioning of a newly discovered element in a group of the PT is not considered or discussed in the published

\footnotetext{
${ }^{*}$ Correspondence: Prof. A. Türler
}

E-mail: andreas.tuerler@dcb.unibe.ch

Department of Chemistry and Biochemistry, University of Bern, Freiestrasse 3, $\mathrm{CH}-3012$ Bern discovery profiles and IUPAC proceeded to place elements with atomic numbers $113,115,117$ and 118 into groups $13,15,17$, and 18 respectively, without giving any further justification. ${ }^{[1,2]}$ Therefore, the $7^{\text {th }}$ period of the PT is now complete and all elements in the PT have proper names, abandoning all provisional names and naming schemes. The names and symbols of all currently known transactinide elements $(Z \geq 104)$ are discussed in section 1.1. and given in Table 1. Also shown for each element is the currently known longest-lived isotope and its half-life. ${ }^{[5]} \mathrm{Up}$ to flerovium, isotopes with half-lives $\geq 1 \mathrm{~s}$ have been observed. The half-life limit for chemical investigations is currently about $1 \mathrm{~s}$, which means that in principle, chemical experiments up to flerovium $(Z=114)$ are possible, and indeed, a number of transactinide elements have already been investigated. ${ }^{[6,7]}$

While, at a first glance, all ambiguities and controversies concerning the PT of the elements seem to be resolved, there remain some issues on further consideration. Interestingly, IUPAC has no recommendation for a specific form of the PT. Indeed, an IUPAC project has been recently initiated to resolve the question whether group 3 consists of the elements Sc, Y, Lu, and Lr, or, of the elements Sc, Y, La, and Ac ${ }^{[8]}$ Furthermore, in the English speaking community, the replacement of the terms 'lanthanides' and 'actinides' with 'lanthanoids' and 'actinoids', respectively, has so far not been widely adopted. ${ }^{[9]}$ On a personal note, I discussed this topic with late Nobel Prize winner G.T. Seaborg. His answer was simply that he, as the discoverer, chose the name 'actinides' and that he was fully aware of the meaning of ending -oid, but that he felt it was too cumbersome to pronounce!

The place an element occupies in the PT is not only defined by its atomic number, i.e. the number of protons in the nucleus, but also by its electronic configuration, which defines its chemical properties. Strictly speaking, a new element is assigned its proper place only after its chemical and atomic properties have been sufficiently investigated. This is not yet the case for a number of elements of the $7^{\text {th }}$ period. No chemical information has been obtained so far for elements meitnerium $(Z=109)$ through roentgenium $(Z=111)$, nihonium $(Z=113)$ and all elements heavier than flerovium $(Z=114)$. Is it then conceivable that the shape of the PT may be altered in the future and that the newly discovered elements are moved away from their current position to different groups? Not very likely, as recent theoretical calculations have shown. ${ }^{[6,10]}$ Nevertheless, strong relativistic effects significantly influence the chemical behaviour of the heaviest elements and, 


\begin{tabular}{|c|c|c|c|c|c|c|c|c|c|c|c|c|c|c|c|c|c|}
\hline 1 & & & & & & & & & & & & & & & & & 18 \\
\hline$\stackrel{1}{\mathrm{H}}$ & 2 & & & & & & & & & & & 13 & 14 & 15 & 16 & 17 & $\begin{array}{l}2 \\
\mathrm{He}\end{array}$ \\
\hline 3 & 4 & & & & & & & & & & & 5 & 6 & 7 & 8 & 9 & 10 \\
\hline Li & $\mathrm{Be}$ & & & & & & & & & & & $B$ & C & $\mathrm{N}$ & 0 & $F$ & $\mathrm{Ne}$ \\
\hline 11 & 12 & & & & & & & & & & & 13 & 14 & 15 & 16 & 17 & 18 \\
\hline $\mathrm{Na}$ & $\mathrm{Mg}$ & 3 & 4 & 5 & 6 & 7 & 8 & 9 & 10 & 11 & 12 & $\mathrm{Al}$ & $\mathrm{Si}$ & $P$ & $S$ & $\mathrm{Cl}$ & $\mathrm{Ar}$ \\
\hline 19 & 20 & 21 & 22 & 23 & 24 & 25 & 26 & 27 & 28 & 29 & 30 & 31 & 32 & 33 & 34 & 35 & 36 \\
\hline $\mathrm{K}$ & $\mathrm{Ca}$ & Sc & $\mathrm{Ti}$ & V & $\mathrm{Cr}$ & $\mathrm{Mn}$ & $\mathrm{Fe}$ & Co & $\mathrm{Ni}$ & $\mathrm{Cu}$ & $\mathrm{Zn}$ & $\mathrm{Ga}$ & $\mathrm{Ge}$ & As & $\mathrm{Se}$ & $\mathrm{Br}$ & $\mathrm{Kr}$ \\
\hline 37 & 38 & 39 & 40 & 41 & 42 & 43 & 44 & 45 & 46 & 47 & 48 & 49 & 50 & 51 & 52 & 53 & 54 \\
\hline $\mathrm{Rb}$ & $\mathrm{Sr}$ & $Y$ & $\mathrm{Zr}$ & $\mathrm{Nb}$ & Mo & Tc & $\mathrm{Ru}$ & $\mathrm{Rh}$ & $\mathrm{Pd}$ & $\mathrm{Ag}$ & $\mathrm{Cd}$ & In & Sn & $\mathrm{Sb}$ & Te & I & $\mathrm{Xe}$ \\
\hline 55 & 56 & $57-71$ & 72 & 73 & 74 & 75 & 76 & 77 & 78 & 79 & 80 & 81 & 82 & 83 & 84 & 85 & 86 \\
\hline Cs & $\mathrm{Ba}$ & La & $\mathrm{Hf}$ & $\mathrm{Ta}$ & W & $\mathrm{Re}$ & Os & Ir & $\mathrm{Pt}$ & $\mathrm{Au}$ & $\mathrm{Hg}$ & $\mathrm{Tl}$ & $\mathrm{Pb}$ & $\mathrm{Bi}$ & Po & At & $\mathrm{Rn}$ \\
\hline 87 & 88 & $89-10$ & 104 & 105 & 106 & 107 & 108 & 109 & 110 & 111 & 112 & 113 & 114 & 115 & 116 & 117 & 118 \\
\hline $\mathrm{Fr}$ & $\mathrm{Ra}$ & Ac & $\mathrm{Rf}$ & $\mathrm{Db}$ & $\mathrm{Sg}$ & $\mathrm{Bh}$ & $\mathrm{Hs}$ & Mt & Ds & $\mathrm{Rg}$ & Cn & $\mathrm{Nh}$ & $\mathrm{FI}$ & Mc & LV & Ts & Og \\
\hline
\end{tabular}

\begin{tabular}{|l|l|l|l|l|l|l|l|l|l|l|l|l|l|l|l|}
\hline \multirow{4}{*}{ Lanthanides } & 57 & 58 & 59 & 60 & 61 & 62 & 63 & 64 & 65 & 66 & 67 & 68 & 69 & 70 & 71 \\
& $\mathrm{La}$ & $\mathrm{Ce}$ & $\mathrm{Pr}$ & $\mathrm{Nd}$ & $\mathrm{Pm}$ & $\mathrm{Sm}$ & $\mathrm{Eu}$ & $\mathrm{Gd}$ & $\mathrm{Tb}$ & $\mathrm{Dy}$ & $\mathrm{Ho}$ & $\mathrm{Er}$ & $\mathrm{Tm}$ & $\mathrm{Yb}$ & $\mathrm{Lu}$ \\
\cline { 2 - 13 } & 89 & 90 & 91 & 92 & 93 & 94 & 95 & 96 & 97 & 98 & 99 & 100 & 101 & 102 & 103 \\
$\mathrm{Ac}$ & $\mathrm{Th}$ & $\mathrm{Pa}$ & $\mathrm{U}$ & $\mathrm{Np}$ & $\mathrm{Pu}$ & $\mathrm{Am}$ & $\mathrm{Cm}$ & $\mathrm{Bk}$ & $\mathrm{Cf}$ & $\mathrm{Es}$ & $\mathrm{Fm}$ & $\mathrm{Md}$ & $\mathrm{No}$ & $\mathrm{Lr}$ \\
\hline
\end{tabular}

Fig. 1. Current periodic table of the elements with IUPAC approved numbering of groups and element symbols.

therefore, experimental chemical investigations of these elements are warranted and of decisive importance. Chemical experiments with heavy and superheavy elements will be discussed in section 3 of this contribution.

This article will conclude with some considerations on the maximum number of elements contained in the PT based on arguments and calculations involving nuclear physics and theoretical chemistry (section 4). As will be shown, since efforts to synthesize elements with $Z=119$ and $Z=120$ have failed so far, hopes to begin and expand the $8^{\text {th }}$ period of the PT have been mitigated. ${ }^{[1-13]}$

\subsection{Names and Symbols of the Elements Most Recently Added to the Periodic Table}

On June 8, 2016, IUPAC released the provisional names for the most recently added elements with $\mathrm{Z}=113,115,117$, and 118 and on November 28, 2016, IUPAC announced the approved names and symbols. ${ }^{[14]}$ Priority of discovery of element 113 was assigned to a team of Japanese scientists from the RIKEN laboratory for observing three decay chains of the nuclide ${ }^{278} 113$ produced in the nuclear fusion reaction ${ }^{209} \mathrm{Bi}\left({ }^{70} \mathrm{Zn}, 1 \mathrm{n}\right)$. The Japanese names for Japan are Nippon and Nihon. The element symbol for 'nipponium' would have created some difficulties, as $\mathrm{Ni}, \mathrm{Np}$, and No are already existing elements. Thus, the name nihonium (Nh) was chosen. Priority for discovery of element 115 was assigned to the Russian-US collaboration for synthesis of the nuclide ${ }^{289} 115$ produced in the reaction ${ }^{243} \mathrm{Am}\left({ }^{48} \mathrm{Ca}, 2 \mathrm{n}\right)$. This nuclide was synthesized for the first time at the Flerov Laboratory of Nuclear Reactions (FLNR) at the Joint Institute for Nuclear Research (JINR) in Dubna, Russia. The name moscowium (Mc) in honor of the city of Moscow was proposed. The city of Dubna, home of FLNR, belongs to the Moscow region. The nuclide ${ }^{293} 117$ was synthesized by a Russian-US collaboration in several experiments employing the reaction ${ }^{249} \mathrm{Bk}\left({ }^{48} \mathrm{Ca}, 4 \mathrm{n}\right)$. The alpha-particle decay chains of ${ }^{293} 117$ proceed through ${ }^{289} 115$ previously synthesized in the reaction ${ }^{243} \mathrm{Am}\left({ }^{48} \mathrm{Ca}, 2 \mathrm{n}\right)$. The element name tennessine (Ts) pays tribute to the fact that the extremely rare target material ${ }^{249} \mathrm{Bk}$ was produced and chemically isolated at the Oak Ridge National
Laboratory (ORNL) employing the high-flux research reactor HIFR in the US state of Tennessee. The ending -ine indicates that tennessine belongs to the group of halogens like fluorine, chlorine, bromine, iodine, and astatine. At this point, the efforts of other laboratories such as Gesellschaft für Schwerionenforschung, Darmstadt, Germany (GSI) and Lawrence Berkeley National Laboratory, Berkeley, US (LBNL) should be acknowledged since they were able to reproduce independently to a large extent the original results of the ${ }^{48} \mathrm{Ca}+{ }^{243} \mathrm{Am}$ and ${ }^{48} \mathrm{Ca}+{ }^{249} \mathrm{Bk}$ reactions. ${ }^{[15-17]}$ Finally, priority of discovery of element 118 was awarded to a Russian-US collaboration employing the reaction ${ }^{249} \mathrm{Cf}\left({ }^{48} \mathrm{Ca}, 3 \mathrm{n}\right)$ to create ${ }^{294} 118$. To date, a total of five atoms from several experimental campaigns were observed. The name of oganesson (Og) honors the outstanding scientific contributions of Russian physicist and leader of the FLNR laboratory Yuri Ts. Oganessian to the discovery of new, superheavy elements. Yu. Ts. Oganessian is thus the second person to be rewarded with the honor of giving his name to a chemical element while still alive. The first one was the Nobel Prize winner G.T. Seaborg. Predictions of the chemical properties of oganesson, the heaviest element in the group of the noble gases are intriguing. ${ }^{[18]}$ One prediction expects oganesson to be the first noble gas with an electron affinity, while another study predicts a condensed phase standard state. ${ }^{[19,20]}$

\section{Synthesis of Heavy and Superheavy Elements}

The heaviest element of natural origin that can be found in abundance in nature is uranium. Trace amounts of neptunium and plutonium were found in naturally occurring uranium ores, where it is formed by irradiation of natural uranium with neutrons followed by beta decay. Neutrons are generated e.g. from $(\alpha, n)-$ reactions or spontaneous fission of uranium isotopes. There are also reports of the detection of relatively long-lived ${ }^{244} \mathrm{Pu}$ in cerium ores. All heavier elements and their isotopes are man-made. The elements americium $(Z=95)$ through fermium $(Z=100)$ can either be bred in high-flux nuclear reactors or are being formed in the intense neutron-flux in explosions of nuclear devices. All trans-fermium elements were synthesized one-atom-at-the-time 
Table 1. Atomic number, element name, chemical symbol, longest-lived known isotope, and half-life of the transactinide elements.

\begin{tabular}{|c|c|c|c|c|}
\hline Atomic number $[Z]$ & Element Name & Chemical Symbol & $\begin{array}{c}\text { Longest-lived } \\
\text { known isotope }\end{array}$ & Half-life $^{[5]}$ \\
\hline 104 & Rutherfordium & $\mathrm{Rf}$ & ${ }^{267} \mathrm{Rf}$ & $1.3 \mathrm{~h}$ \\
\hline 105 & Dubnium & $\mathrm{Db}$ & ${ }^{268} \mathrm{Db}$ & $28.3 \mathrm{~h}$ \\
\hline 106 & Seaborgium & $\mathrm{Sg}$ & ${ }^{269} \mathrm{Sg}$ & $3.1 \mathrm{~m}$ \\
\hline 107 & Bohrium & $\mathrm{Bh}$ & ${ }^{270} \mathrm{Bh}$ & $61 \mathrm{~s}$ \\
\hline 108 & Hassium & $\mathrm{Hs}$ & ${ }^{269} \mathrm{Hs}$ & $9.7 \mathrm{~s}$ \\
\hline 109 & Meitnerium & $\mathrm{Mt}$ & ${ }^{278} \mathrm{Mt}$ & $12.7 \mathrm{~s}$ \\
\hline 110 & Darmstadtium & $\mathrm{Ds}$ & ${ }^{281} \mathrm{Ds}$ & $100 \mathrm{~s}$ \\
\hline 111 & Roentgenium & $\mathrm{Rg}$ & $28 \mathrm{~s}$ \\
\hline 112 & Copernicium & $\mathrm{Cn}$ & ${ }^{282} \mathrm{Rg}$ & $9.5 \mathrm{~s}$ \\
\hline 114 & Nihonium & $\mathrm{Nh}$ & ${ }^{285} \mathrm{Cn}$ & $1.9 \mathrm{~s}$ \\
\hline 115 & Flerovium & $\mathrm{Fl}$ & ${ }^{286} \mathrm{Nh}$ & $650 \mathrm{~ms}$ \\
\hline 116 & Moscovium & $\mathrm{Mc}$ & ${ }^{289} \mathrm{Lv}$ & $57 \mathrm{~ms}$ \\
\hline 117 & Livermorium & $\mathrm{Lv}$ & ${ }^{290} \mathrm{Mc}$ & $51 \mathrm{~ms}$ \\
\hline
\end{tabular}

in nuclear fusion reactions and are available only in non-weighable amounts.

Superheavy nuclei with $Z \geq 104$ owe their existence exclusively to nuclear shell effects. It has been shown that higher order deformations of superheavy nuclei lead to significant gains of shell correction energy compared to the simple liquid drop model of the nucleus and therefore result in substantial increases of the fission barrier.[21] Also, certain lighter nuclei exhibit especially high binding energies of the nucleons, which are associated with the so-called magic numbers of protons and neutrons. These socalled stable doubly magic nuclei are ${ }^{4} \mathrm{He},{ }^{16} \mathrm{O},{ }^{40} \mathrm{Ca},{ }^{48} \mathrm{Ca}$, and ${ }^{208} \mathrm{~Pb}$. Modern nuclear shell models predict two islands of increased stability past ${ }^{208} \mathrm{~Pb}$. A deformed shell closure is reached at $Z=108$ and $N=162\left({ }^{270} \mathrm{Hs}\right)$ while the so-called island of stability of superheavy nuclei is centered around $Z=114$ and $N=184$. ${ }^{[21]}$ Due to the neutron-richness of this latter doubly magic nucleus, all combinations of stable nuclei lead to landing points east of $N$ $=184$, so to say on the shores of the island of stability (Fig. 2).

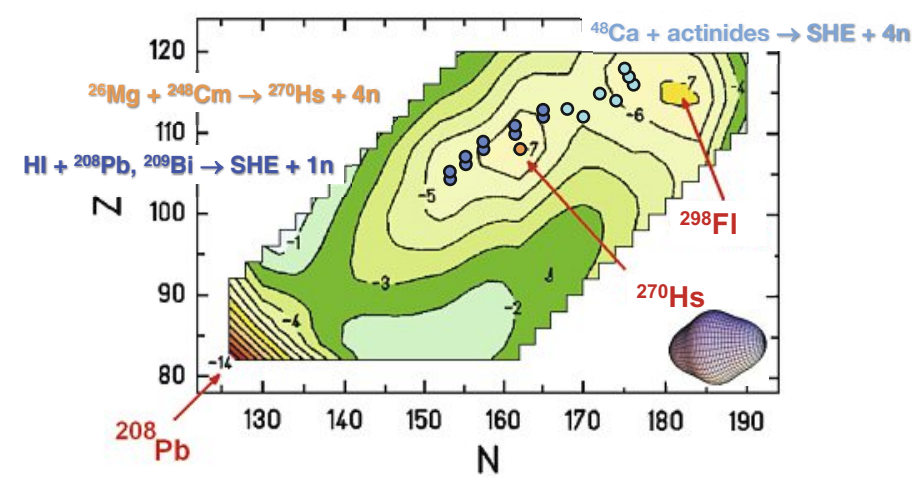

Fig. 2. Shell correction energies for nuclides with $Z \geq 82$ and $N \geq 126$. Two regions of enhanced nuclear stability are visible at ${ }^{208} \mathrm{~Pb},{ }^{270} \mathrm{Hs}$ and ${ }^{298} \mathrm{FI}$. Landing points of different nuclear fusion reactions are indicated. Figure adopted from ref. [21].

\subsection{Nuclear Reactions Leading to the Synthesis of Heavy and Superheavy Elements}

In nuclear fusion reactions, a projectile nucleus is fused with a target nucleus to form a new heavy compound nucleus whose atomic number is the sum of the atomic numbers of projectile and target. The projectiles are accelerated by means of a particle accelerator (large cyclotron or linear accelerator) to energies surpassing the Coulomb barrier. This way, the nuclear matter of projectile and the target come into contact and in rare cases, a compound nucleus is formed. As in chemical reactions, nuclear fusion of heavy nuclei is mostly exoergic, meaning that the fused compound nucleus carries a high excitation energy in the form of rotational- and vibrational energy as well as in the form of excitation energy. In most cases, the high excitation energy leads to prompt fission of the compound nucleus and the fusion product is lost. Rarely, excitation energy is dissipated by the evaporation of neutrons. Each evaporation step competes with prompt fission. After the evaporation of 2 to 5 neutrons, the compound nucleus has cooled sufficiently to dissipate further energy by the emission of gamma-rays (Fig. 3). The newly formed nucleus of a heavy element now survives sufficiently long to allow its detection by radioactive decay. Prominent decay modes of heavy nuclei are alpha-particle emission or spontaneous fission. The probability of forming a heavy nucleus is governed by the fusion probability of two nuclei and the survival probability of the compound nucleus. Both probabilities are strongly dependent on the choice of projectile- and target nucleus. A strong mass asymmetry in the entrance channel, i.e. the fusion of a light projectile with a heavy target nucleus strongly enhances the fusion probability. However, such combinations are characterized by large reaction Q-values (large mass excess) and therefore by low survival probabilities, requiring the evaporation of 5 to 6 neutrons to cool the compound nucleus. Such reactions are termed 'hot fusion reactions' characterized by excitation energies of 50-60 MeV. More symmetric combinations are hampered by low fusion probabilities, but once a compound nucleus is formed, the excitation energy is much low- 


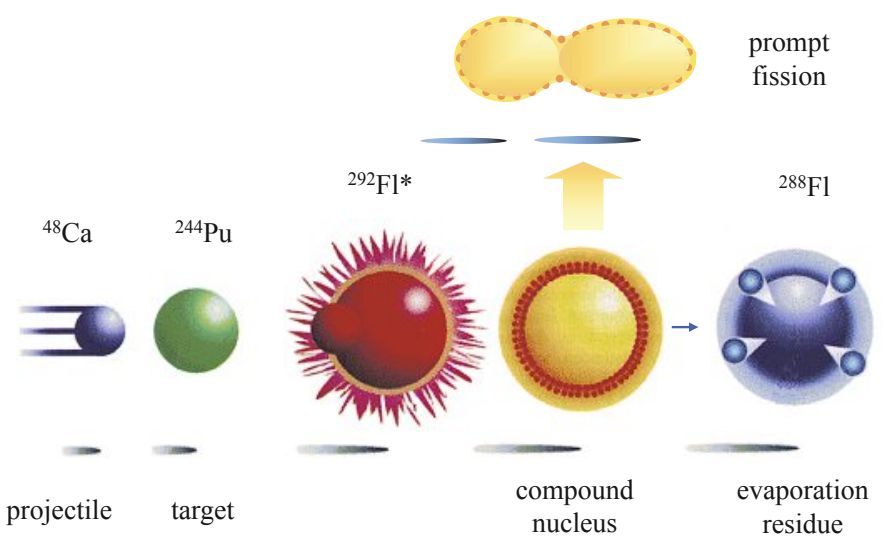

Fig. 3. Schematic representation of a nuclear fusion reaction. A projectile nucleus fuses with a target nucleus to create a highly excited compound nucleus, which mostly disintegrates by prompt fission into two fragments. Infrequently, several neutrons are evaporated to produce an observable evaporation residue.

er, of the order of 10-20 MeV, requiring the evaporation of only 1 to 2 neutrons. Such reactions are termed 'cold fusion reactions'.

The use of doubly magic nuclei as projectile or target (or both) significantly cools the compound system due to the large energy consumed in breaking up the magic configurations. Therefore, projectile target combinations using ${ }^{208} \mathrm{~Pb}$ as target material led to the synthesis and discovery of elements bohrium $(Z=107)$ through nihonium $(Z=113)$. Because the production rates finally drop down to few detected atoms per year of beam time, this route is not used to synthesize even heavier elements.

A more productive route, leading to more neutron-rich heavy nuclei was discovered in using doubly magic ${ }^{48} \mathrm{Ca}$ as projectile and neutron-rich heavy actinide nuclei as target materials. These reactions were termed 'warm fusion reactions' leading to the evaporation of 2-4 neutrons. Using this strategy, new neutronrich nuclei of elements hassium $(Z=108)$ up to oganesson $(Z$ $=118$ ) were synthesized and detected. This approach was pioneered at the Flerov Laboratory of the Joint Institute for Nuclear Research (JINR) in Dubna, Russia. All major experiments leading to the discovery of elements flerovium, moscovium, livermorium, tennessine, and oganesson, were conducted in Dubna in close collaboration with groups from Lawrence Livermore National Laboratory (LLNL) and Oak Ridge Nationl Laboratory (ORNL) from the United States. ${ }^{[5]}$ The US laboratories contributed target technologies and tens of milligrams of the extremely valuable, very exotic actinide target materials, but also know-how and detector technologies.

\subsection{Isolation of Single Atoms of Heavy and Superheavy Elements}

Once a heavy element has been synthesized in a nuclear fusion reaction, it has to be immediately separated from a plethora of other reaction products so that it can be identified via its radioactive decay. Nowadays, this is accomplished within few microseconds with the aid of kinematic separators. ${ }^{[22]}$ Due to the kinetic energy imparted by the projectile, the fusion-evaporation residue carries the momentum of the beam and is ejected from the target nucleus with a well-defined velocity. Thus, the newly formed nucleus carries a distinctive charge and kinetic energy. Presently, kinematic separators are filtering reaction products in flight by velocity, mass, or energy or mass/charge ratio. The velocity filter SHIP at GSI Darmstadt, which is a vacuum separator that consists of two symmetrically arranged Wien-filters with spatially separated electrostatic and magnetic dipoles has proved extremely successful. An additional small dipole magnet at the end of the flight path bends the product beam out of the direct line of sight of the target position and strongly reduces unde- sired background. For ${ }^{48} \mathrm{Ca}$-induced reactions the transmission through the separator was reported to be about $20 \%$. Gas-filled magnetic dipole separators have significantly higher efficiency. Evaporation residues can be spatially separated from the incoming beam and from transfer-reaction products in a gas-filled dipole magnet making use of a charge focusing effect. The recoiling reaction products constantly change their charge state in collisions with a dilute gas (usually $\mathrm{H}_{2}$ or $\mathrm{He}$ ) resulting in an average charge state. The magnetic rigidities of the beam and other reaction products are significantly different, which allows a separation of the product beam. The transmission is about $40 \%$ for the DGFRS (Dubna Gas-Filled Recoil Separator) and up to about $50 \%$ for TASCA (TransActinide Separator and Chemistry Apparatus) at GSI. The gas-filled separators BGS (Berkeley Gasfilled Separator) or GARIS (Gas-filled Recoil Ion Separator) at RIKEN, Japan have similar or even better transmission.

\subsection{Identification of Single Atoms of Heavy and Superheavy Elements}

Fortunately, all nuclei so far observed heavier than ${ }^{208} \mathrm{~Pb}$ are radioactive. Heavy and superheavy nuclei mostly decay by the emission of alpha-particles $\left({ }^{4} \mathrm{He}^{2+}\right)$ or by spontaneous fission. Therefore, such nuclei can be detected very sensitively down to the single atom level. Superheavy nuclei are frequently characterized by decay chains, the rapid emission of several alpha particles in a short time period and often terminated by spontaneous fission. These signatures are unique. In order to detect such signatures, highly efficient particle detectors have been developed that even offer spatial resolution. An evaporation residue travelling through the separator is implanted into a position-sensitive silicon strip detector, where the implantation signal and the position are registered. Usually, after a short time period, a first alpha particle is emitted, followed by one or several daughter alpha particles and often terminated by spontaneous fission of high energy. If this sequence occurs in one pixel of the silicon detector, and all alpha particle energies are within certain energy windows, the probability that this sequence is of random origin is very small and the decay chain of a superheavy nucleus has been observed. Often the lower members of the decay chain are well known from previous experiments and thus the atomic number of the decaying nucleus can be pinpointed. Unfortunately, alpha particles can in some cases also leave the silicon detector in a backwards direction since the implantation depth is very shallow. Such alpha particles can be registered with side detectors that surround the stop detector upstreams and form a well. If now a low energy signal is detected in the stop detector and the remainder in coincidence in one of the side detectors, the original alpha particle decay energy can be reconstructed. This applies also to spontaneous fission, where one of the fragments has a relatively high probability of being emitted in a backward (upstream) direction. A schematic of such a detector arrangement is shown in Fig. 4. In addition, time of flight detectors are also installed upstream to allow to distinguish signals in the stop detector that arise from implanted nuclei from those that arise from alpha particle decays. All events are stored in list mode with a precise time stamp. A search experiment for a new superheavy element generates several tens of terabytes of data, containing one, maybe two decay sequences. Therefore, the analysis of such data is very time consuming and resembles the search for a needle in a haystack. In recent years, the detector box was also surrounded with a number of Ge-crystals to perform gammaand X-ray spectroscopy. The detection of characteristic X-rays would constitute the ultimate tool in the identification of the atomic number of the observed superheavy nuclei. However, so far no X-rays have been observed, ${ }^{[15,17]}$ probably due to the fact that they are highly converted and thus their emission probability is very low. 


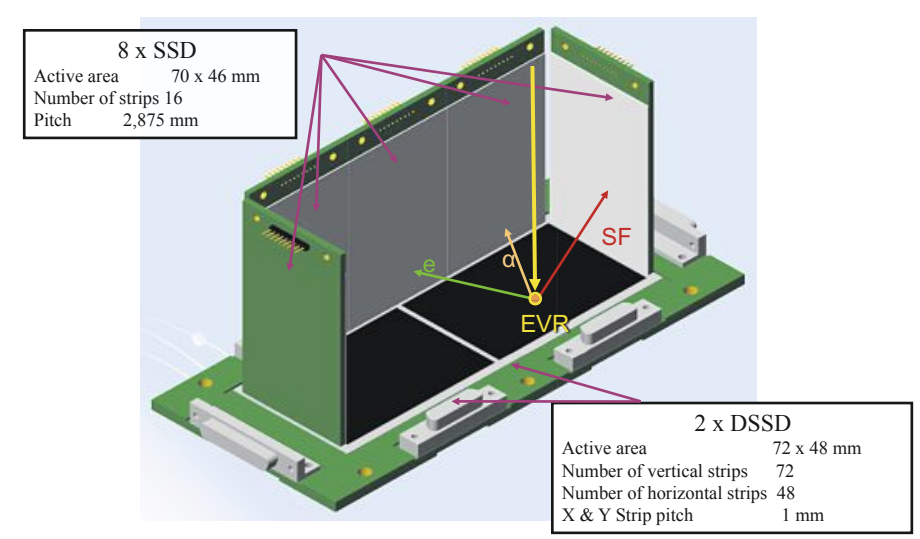

Fig. 4. Detector assembly for the identification of superheavy nuclei. The stop detector consists of two double-sided strip detectors (DSSD) of 72 x $48 \mathrm{~mm}$ area and $1 \mathrm{~mm}$ pitch size. The side detectors facing upstream are single strip detectors (SSD) of $70 \times 46 \mathrm{~mm}$ size and $2.875 \mathrm{~mm}$ strip width. The total efficiency to detect a particle emitted from an evaporation residue implanted into the stop detector amounts to about $72 \%$.

\section{Chemical Experiments with Heavy and Superheavy Elements}

For a chemist the most thrilling but also the most challenging task is to chemically characterize a newly discovered chemical element and to pinpoint its position in the PT based on its properties and its electronic structure. The fact that only single, very short-lived atoms are available for experiments makes this task extremely difficult. Therefore, heavy and superheavy element chemistry is limited to the measurement of only a few basic chemical properties on a 'one-atom-at-the-time' level. Nevertheless, such experiments already deliver a wealth of information and allow assessing the influence of relativistic effects, which play a dominant role at the bottom end of the PT. A crucial contribution is made by theoretical chemistry. Thanks to ever-increasing computing power, very detailed and accurate atomic- and molecular calculations are now becoming possible, even including quantum electrodynamic (QED) effects. ${ }^{[23]}$ Also, sophisticated calculations describing the interaction with the surface of an extended slab involving more than hundred atoms are feasible. ${ }^{[10]}$ In the following, recent accomplishments in heavy and superheavy element chemistry will be discussed; reviews of older data are given in refs. [6] and [7].

Very recently, in a number of experiments the first ionization potential of the heaviest actinides including Lr was measured using a surface ionization technique and also, in one case, laser spectroscopy. [23,24] Sato et al. determined the first ionization potentials of Fm, Md, No, and Lr. ${ }^{[25]}$ The authors summarized their work as follows: "Similar to the well-established behavior for the lanthanides, the IP values of the heavy actinides up to No increase with filling up the $5 \mathrm{f}$ orbital, while that of $\mathrm{Lr}$ is the lowest among the actinides. These results clearly demonstrate that the $5 \mathrm{f}$ orbital is fully filled at No with the $[\mathrm{Rn}] 5 \mathrm{f}^{14} 7 \mathrm{~s}^{2}$ configuration and that Lr has a weakly bound electron outside the No core. In analogy to the lanthanide series, the present results unequivocally verify the actinide series ends with Lr.".[25] The technique employed holds some promise that also the IP of the first transactinide element Rf may be measured but it will prove to be a 'tour de force'. Chhetri et al. determined the first ionization potential of the element nobelium using laser spectroscopy with unprecedented precision to be $6.62621 \pm 0.00005 \mathrm{eV}$. Such precision measurements offer benchmarks for state-of-the art atomic calculations including relativistic- and QED effects. ${ }^{\text {[24] }}$

The early transactinide elements have already been investigated by chemical means. Elements $\mathrm{Rf}$ through $\mathrm{Sg}$ have been investigated in the aqueous phase. ${ }^{[6]}$ In the gas-phase, the body of data is even more extensive, where experiments with elements Rf through Hs have been carried out. ${ }^{[6,7]]}$ Also, first experiments were conducted with $\mathrm{Cn}, \mathrm{Nh}$, and $\mathrm{Fl}$ in elemental form. ${ }^{[7,26]}$ The prevalence of experiments using gas-phase chemical separations is due to the fact that production of transactinide elements at accelerators usually implies a thermalization of the primary products in a gas, a so-called recoil chamber, after kinematic separation by a recoil separator. ${ }^{[6,7]}$ It is rather straightforward to connect such a recoil chamber to a gas chromatographic separation system and to condense the separated volatile species for alpha-particle spectroscopy and spontaneous-fission detection. Lately, this technique was employed to investigate the volatility of seaborgium hexacarbonyl $\mathrm{Sg}(\mathrm{CO})_{6}$ with a set-up shown in Fig. 5.[27] The ion beam from the RILAC accelerator passed through the rotating target assembly. The primary beam was separated from the evaporation residues within the field of the first, gas-filled dipole magnet (D). Evaporation residues were focused by two quadrupole magnets $(\mathrm{Q})$ and deflected to the focal plane by a second dipole magnet. At the focal plane, evaporation residues of Mo, W, and $\mathrm{Sg}$ passed a thin Mylar window and entered the Recoil Transfer Chamber RTC, which was flushed with a $\mathrm{He} / \mathrm{CO}$ mixture. In this mixture, the evaporation residues were thermalized, converted to volatile carbonyl complexes and transported with the gas stream through a 10-m long perfluoroalkoxy Teflon capillary to the thermochromatographic detector array COMPACT, along which a negative temperature gradient was established with a liquid nitrogen cryostat. Volatile species deposited on the detector surface at a specific temperature and were identified through the detection of their nuclear decay. The observed deposition pattern is shown in Fig. 6. Using a microscopic model describing the movement of a molecule along the thermochromatographic array, the respective adsorption enthalpies of $\mathrm{Mo}(\mathrm{CO})_{6}, \mathrm{~W}(\mathrm{CO})_{6}$ and $\mathrm{Sg}(\mathrm{CO})_{6}$ could be evaluated. ${ }^{[27]}$

Concerning the chemical properties of $\mathrm{Cn}$ and $\mathrm{Fl}$, no conclusive verdict has been reached so far. ${ }^{[26]}$ It appears safe to say that indeed both elements are rather volatile and inert and do not interact strongly with other elements. While $\mathrm{Hg}$, the lighter homologue of $\mathrm{Cn}$, is a liquid at room temperature and rather volatile, it still interacts strongly with Au surfaces. As predicted by theoretical calculations, $\mathrm{Cn}$ seems to exhibit a much weaker tendency to interact with Au. Experimental data for Fl still lacks statistics, and no conclusions concerning the volatility of Fl relative to $\mathrm{Cn}$ can be drawn at this time. Currently, there is only one publication available dealing with the experimental investigation of Nh. ${ }^{[28]} \mathrm{A}$ critical discussion of this experiment is given in ref. [7].

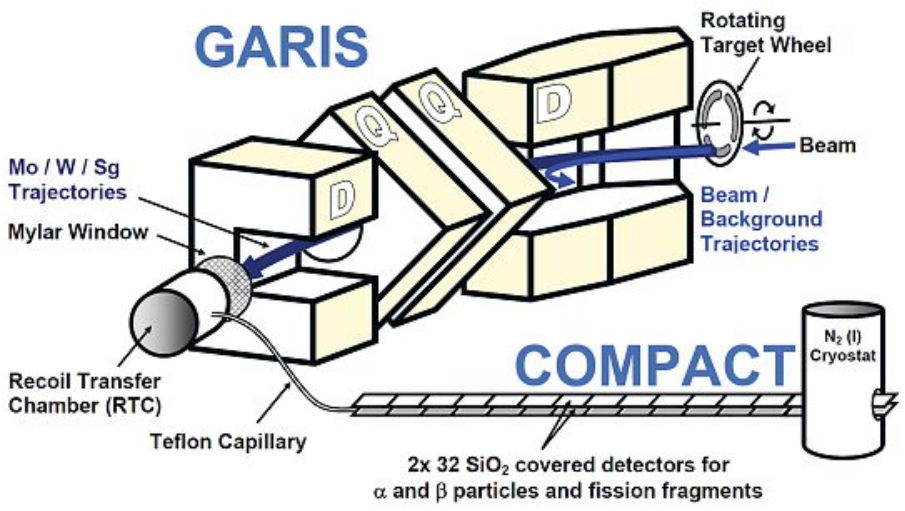

Fig. 5. Schematic drawing of the experimental setup for the synthesis and identification of $\mathrm{Sg}(\mathrm{CO})_{6}$. For a detailed description see text. Reprinted with permission from ref. [27]. Copyright (C 2014 American Association for the Advancement of Science. 


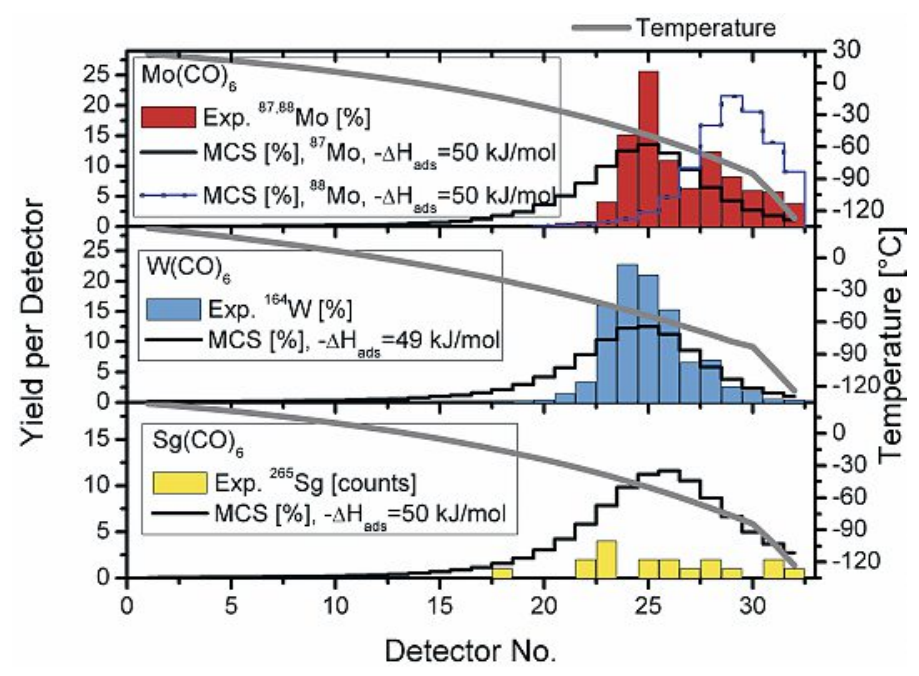

Fig. 6. Deposition patterns of volatile group-6 hexacarbonyl complexes in a thermochromatographic temperature gradient. The adsorption enthalpies deduced indicate that group- 6 hexacarbonyls, including Sg, show an almost identical volatility. Reprinted with permission from ref. [27]. Copyright (C) 2014 American Association for the Advancement of Science.

\section{Natural Limits to the Expansion of the Periodic Table}

Chemical and nuclear physics arguments limit the maximum size of the PT of the elements. The shortest time period an element needs to exist to be recognized as a member of the PT was defined as $>10^{-14} \mathrm{~s}$, which is the time needed for the formation of an electron shell. However, inflight separation with a kinematic separator requires times of the order of microseconds. The recipe so far successful to synthesize superheavy elements involved the fusion of doubly magic ${ }^{48} \mathrm{Ca}$ with an actinide target. Unfortunately, only few micrograms or even nanograms of Es and Fm respectively will be available in the near future. Thus, the synthesis of elements 119 and 120 has to rely on new, less favorable combinations of projectile and target. So far, several experiments have failed to find evidence for the synthesis of a new element. ${ }^{[11]}$ Nevertheless, theoretical predictions of nuclear fusion reactions indicate that the synthesis of elements 119 and 120 lies within reach of present day technologies. Concerning the synthesis of elements beyond $Z$ $=120$, the outlook is rather sobering. The half-life limit of microseconds may be reached rather quickly. According to theoretical predictions all isotopes of elements 119-124 have half-lives of less than one millisecond. ${ }^{[29]}$

From the chemical point of view, the boundary conditions are less stringent. The number of electrons that can be arranged around a nucleus is limited. Modern relativistic electronic structure theory that takes into account quantum electrodynamic effects (QED) predicts this to happen at element $Z=173$, where the energy of the $1 \mathrm{~s}$ electron falls into the negative energy continuum. ${ }^{[30]}$

\section{Outlook and Conclusions}

The expansion of the PT will continue at a reduced pace. The growth of the number of transuranium elements was characterized by periods of rapid growth, followed by periods of stagnation, as new concepts or new technologies had to be developed. The discovery of new elements will most likely happen in the domain of nuclear physics. In order to increase the sensitivity of future experiments by one order of magnitude, several technological improvements have to be realized. New dedicated accelerators will deliver higher beam intensities requiring target technologies capable of absorbing the beam power. New recoil separators with increased transmission and better suppression of unwanted reaction products will provide an increase in sensitivity. All this will be realized in the superheavy element factory currently under construction in Dubna, Russia. Thus, the first synthesis of elements 119 and 120 and the opening of the $8^{\text {th }}$ period of the PT should come within reach in the next couple of years. Synthesis of elements past atomic number 120 , however, will remain a daunting task and discovery of the first superactinide element with $Z=$ 122 may one day become possible, but very likely not in the near future.

Chemical experiments with transactinide elements will also profit from the available beam time offered by the superheavy element factory in Dubna. First conclusive chemical experiments with $\mathrm{Nh}$ and $\mathrm{Fl}$ are within reach, whereas due to their chemical behaviour and/or the short half-lives, chemical experiments with Mt, Ds, and Rg, or the trans-flerovium elements remain very challenging. Nevertheless, as recent experiments have shown, experimental investigations of the heaviest elements in the PT is of fundamental interest to chemists and physicists alike and provide deep insights into the structure and ordering principles governing the PT.

Received: January 30, 2019

[1] P. J. Karol, R. C. Barber, B. M. Sherrill, E. Vardaci, T. Yamazaki, Pure Appl. Chem. 2016, 88, 139.

[2] P. J. Karol, R. C. Barber, B. M. Sherrill, E. Vardaci, T. Yamazaki, Pure Appl. Chem. 2016, 88, 155.

[3] J. Corish, Chem. Int. 2016, March-April, 9.

[4] W. H. Koppenol, J. Corish, J. García-Martínez, J. Meija, J. Reedijk, Pure Appl. Chem. 2016, 88, 401.

[5] Y. T. Oganessian, A. Sobiczewski, G. M. Ter-Akopian, Phys. Scr. 2017, 92, 023003 .

[6] A. Türler, V. Pershina, Chem. Rev. 2013, 113, 1237.

[7] A. Türler, R. Eichler, A. Yakushev, Nuclear Phys. A 2015, 944, 640.

[8] E. Scerri, Chem. Int. 2016, March-April, 22.

[9] E. Fluck, Pure Appl. Chem. 1988, 60, 431.

[10] V. Pershina, Nuclear Phys. A 2015, 944, 578.

[11] C. E. Düllmann, EPJ Web of Conferences 2017, 163, 00015.

[12] Y. T. Oganessian, V. K. Utyonkov, Y. V. Lobanov, F. S. Abdullin, A. N. Polyakov, R. N. Sagaidak, I. V. Shirokovsky, Y. S. Tsyganov, A. A. Voinov, A. N. Mezentsev, V. G. Subbotin, A. M. Sukhov, K. Subotic, V. I. Zagrebaev, S. N. Dmitriev, R. A. Henderson, K. J. Moody, J. M. Kenneally, J. H. Landrum, D. A. Shaughnessy, M. A. Stoyer, N. J. Stoyer, P. A. Wilk, Phys. Rev. C 2009, 79, 024603.

[13] S. Hofmann, S. Heinz, R. Mann, J. Maurer, G. Münzenberg, S. Antalic, W. Barth, H. G. Burkhard, L. Dahl, K. Eberhardt, et al., Eur. Phys. J. A 2016 52, 180.

[14] L. Öhrström, J. Reedijk, Pure Appl. Chem. 2016, 88, 1225.

[15] D. Rudolph et al., Phys. Rev. Lett. 2013, 111, 112502.

[16] J. Khuyagbaatar et al., Phys. Rev. Lett. 2014, 112, 172501.

[17] J. M. Gates et al., Phys. Rev. C 2015, 92, 021301(R).

[18] P. Jerabek, B. Schuetrumpf, P. Schwerdtfeger, W. Nazarewicz, Phys. Rev. Lett. 2018, 120, 053001.

[19] E. Eliav, U. Kaldor, Y. Ishikawa, P. Pyykkö, Phys. Rev. Lett. 1996, 77, 5350.

[20] C. S. Nash, J. Phys. Chem. A 2005, 109, 3493.

[21] A. Sobiczewski, I. Muntian, Z. Patyk, Phys. Rev. C 2001, 63, 034306.

[22] Ch. E. Düllmann, Nucl. Instrum. Meth. B 2008, 266, 4123.

[23] T. K. Sato et al., Nature 2015, 520, 209.

[24] P. Chhetri et al., Phys. Rev. Lett. 2018, 120, 263003

[25] T. Sato, et al., J. Am. Chem. Soc. 2018 140, 14609.

[26] A. Yakushev, R. Eichler, EPJ Web of Conferences 2016, 131, 07003.

[27] J. Even et al., Science 2014, 345, 1491.

[28] S. N. Dmitriev, N. V. Aksenov, Y. V. Albin, Mendeleev Commun. 2014, 24 253.

[29] T. L. Zhao, X. J. Bao, Phys. Rev. C 2018, 98, 064307.

[30] P. Indelicato, J. Bieron, P. Jonsson, Theor. Chem. Acc. 2011, 129, 495. 